\title{
Effects of additives on the activity of a covalently immobilised lipase in organic media
}

\author{
J.M.S. Rocha *, M.H. Gil, F.A.P. Garcia \\ Chemical Engineering Department, University of Coimbra, 3000 Coimbra, Portugal
}

Received 6 January 1998; accepted 26 February 1998

\begin{abstract}
Lipase from Mucor miehei was covalently immobilised onto the graft copolymer poly(ethylene)-g.co-hydroxyethyl methacrylate (PE-g.co-HEMA), partially hydrolysed, via a spacer arm of 1,6-diaminohexane activated with glutaraldehyde. To improve the lipolytic activity of the immobilised lipase (for the synthesis of isoamyl-caprylate, as a model), the effect of several additives was investigated. Polyethylene glycol (PEG), glutaraldehyde, organic solvents and buffers, were added during the immobilisation procedure and their effects are reported and compared with the behaviour of the lipolytic preparation without pre-treatment. An increase of $40-100 \%$ in the activity was obtained when small quantities of PEG 2000 and glutaraldehyde (used also as an activator of the spacer arm) were added. The activity had a maximum when the $\mathrm{pH}$ of the lipase attachment solution was 7.2 and buffered with phosphate. The effect of the aggregation level of biocatalyst particles on the amount of water retained, as well as the effect of the immobilisation on solid supports on the stability to organic solvents, is also reported. (C) 1998 Elsevier Science B.V. All rights reserved.
\end{abstract}

Keywords: Lipase; Additives; Biocatalysis; Organic media

\section{Introduction}

Due to their good mechanical strength and chemical functionality new synthetic polymers have great potential as support materials for enzyme immobilisation. The graft copolymer PEg.co-HEMA, in particular, with a backbone of

\footnotetext{
* Corresponding author. Tel.: + 351 39828392; fax: + 351 39841138; e-mail: jrocha@eq.uc.pt
}

poly(ethylene) and side chains of poly(hydroxyethyl methacrylate), partially hydrolysed, is a good material for lipase immobilisation, due to the ability for creating a microenvironment favourable for the catalysis of synthesis reactions with hydrophobic substrates. Moreover, it shows high affinity for the lipase from crude aqueous solutions and high levels of activity retention after immobilisation, with a successful application as biocatalyst in the synthesis of esters from car- 
boxylic acids and in the interesterification of tryglicerides (Rocha, 1996).

Possible ways to increase the lipolytic activity include the manipulation of the lipase structure, the method of immobilisation, the medium conditions or the use of additives. The influence of additives in enzymatic activity is not well understood. Most of the studies that have been reported involve the co-immobilisation of the additive in a simple adsorption process (or slightly modified by precipitation of the enzyme, by evaporation of the water or by lyophilization) and its effects are attributed to, (i) enzyme protection from inactivation during the immobilisation step (Wehtje et al., 1993; Triantafyllou et al., 1995); (ii) retention of the water shell around the catalyst (Adlercreutz, 1993; Triantafyllou et al., 1995), or (iii) dispersing effects of the enzyme molecules and facilitated mass transport when such additives are used as immobilisation matrices (Triantafyllou et al., 1997). Sometimes, the role of these additives (proteins, saccharides, polyols and salts), is performed by the inert impurities included in crude enzyme solutions (Yamane et al., 1990).

Beyond the aforementioned indirect effects, other and more direct effects (conformational or ionic changes in the molecular lipase structure) on the activity and the stability of lipases must be investigated. The additive can be present or absent in the medium during the reaction and sometimes its contact with the support or with the lipase is sufficient. The presence of the additive in the enzymatic reaction medium can also have negative effects on the reaction kinetics or on the mass transfer resistances. Their contact with ei-

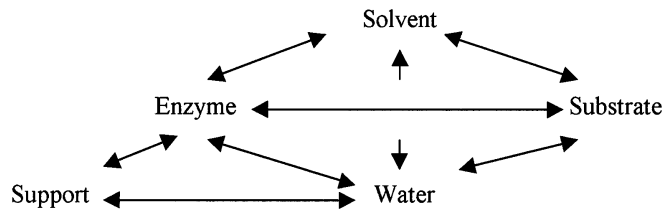

Fig. 1. Mutual relationships between the several components involved in a synthesis biocatalytic reaction in the presence of organic solvents.

ther the support or with the lipase can be done before, during or after the immobilisation.

In this work the effect of some additives such as polyethylene glycol (PEG), glutaraldehyde, organic solvents and buffers, during the immobilisation procedure is reported and compared with the behaviour of the lipolytic preparation with no pre-treatment.

\section{Experimental}

\subsection{Biocatalyst}

The support was a PE-g.co-HEMA with $116 \%$ grafting, $4 \mathrm{~h}$ of hydrolysis by refluxing it in $1 \mathrm{M}$ $\mathrm{NaOH}$ and of an average particle size in the range $300-350 \mu \mathrm{m}$.

The enzyme was a fungal lipase (from Mucor miehei ) as crude solution, (Lipozyme ${ }^{\circledR} 10000$ L), kindly donated by Novo Nordisk, Denmark.

The immobilisation procedure was a three-sequential steps process, as described by Rocha et al. (1994). In the 1st step the $-\mathrm{COOH}$ groups (in $50 \mathrm{mg}$ of copolymer) were activated with carbodiimide and spacer arms of 1,6-diaminohexane were

Table 1

Effect of the water activity $\left(a_{\mathrm{w}}\right)$ on the immobilised lipase activity (synthesis of $n$-octyl oleate) and on the water mass content of the biocatalyst

\begin{tabular}{lcccc}
\hline & Set & \multicolumn{2}{l}{ Water activity $\left(a_{\mathrm{w}}\right)$} & \\
\cline { 4 - 5 } & & $a_{\mathrm{w}}=0.32$ & $a_{\mathrm{w}}=0.86$ & $a_{\mathrm{w}}=1.00$ \\
\hline Biocatalyst water content (\%) & $1 \mathrm{st}$ & 0.0 & 9.3 & 62.5 \\
& $2 \mathrm{nd}$ & 3.8 & 9.9 & 127.5 \\
Relative initial rate (\%) & $1 \mathrm{st}$ & 61.6 & 82.2 & 100 \\
& 2nd & 78.7 & 100 & 41.7
\end{tabular}




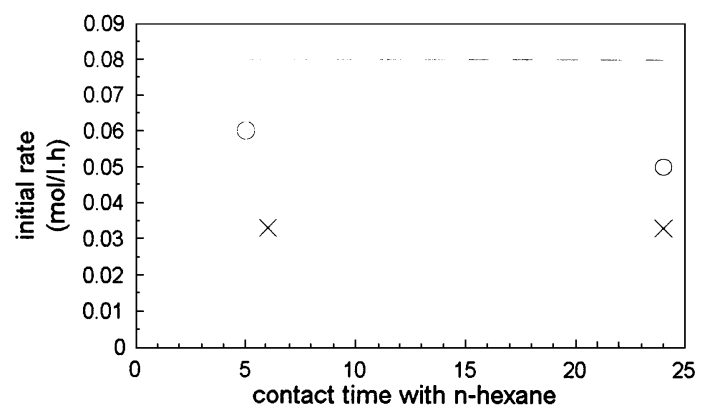

(h)

(a)

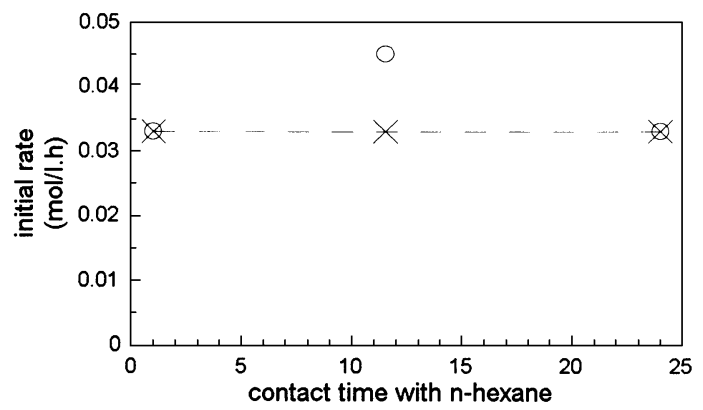

(h)

(b)

Fig. 2. (a) Influence of the contact time of free lipase with $n$-hexane, under strong mixing conditions, before the immobilisation step, on the esterification activity of the hydrated lipolytic preparation. (b) Influence of the contact time of the dehydrated lipolytic preparation with $n$-hexane, under low stirring conditions, on the initial esterification activity. In both cases the horizontal broken line represents the activity of the immobilised biocatalyst without previous contact with $n$-hexane: $(-\circ-) T=12^{\circ} \mathrm{C} ;(-\times-)$ $T=37^{\circ} \mathrm{C}$.

inserted; the 2nd step was the activation of $-\mathrm{NH}_{2}$ groups with glutaraldehyde; the 3rd step was the immobilisation of the lipase by adding $200 \mu 1$ of crude lipase solution in $6 \mathrm{ml}$ of phosphate buffer $(\mathrm{pH} 7,100 \mathrm{mM})$ to the activated copolymer.

\subsection{Esterification reaction}

The esterification reaction was a direct reaction of $5 \mathrm{ml}$ of isoamyl alcohol (3-methylbutanol-1, from Merck) and $0.8 \mathrm{ml}$ of caprylic acid (octanoic acid, from Merck) to produce isoamyl caprylate, in the absence of organic solvents. The reaction was started by the addition of the biocatalyst particles in a batch reactor under magnetic stirring at $T=37^{\circ} \mathrm{C}$; the ester formation profile was evaluated by titration of the residual fatty acid in the reaction mixture along the time; the lipolytic activity was determined by the initial rate of reaction $\left(\mathrm{mol} \mathrm{l}^{-1} \mathrm{~h}^{-1}\right)$.

\section{Results and discussion}

\subsection{The role of water}

The heterogeneity of biocatalytic systems in organic media implies mutual relationships among the different components of the systems, as shown in Fig. 1. When a biocatalyst, or a biocatalyst preparation, is selected for a given type of reaction, the kind of organic solvent, the water content and the solubility of substrates and products, must all be evaluated or optimised. Simple dual relationships between two components may lead to global misinterpretations.

Water is perhaps the most important 'additive' when biocatalysis is performed in organic media. It is well known that a minimum amount of water, which depends on the type of organic solvent used and the characteristics of the immobilisation support, is absolutely necessary to the solvation of either the enzyme or the substrates and products. However, an excess of water will favour hydrolysis instead of synthesis reactions. Thus a compromise must be established and an optimum amount of water must be determined, and this can be better accomplished with immobilised biocatalysts.

The 'water activity' concept is, of course, a main tool in the analysis of biocatalysis in organic medium, and to a certain extent it caused the 'water content' to become an obsolete concept. In our view both concepts are important. When different solvents and substrates are equilibrated in atmospheres with the same water activity, to be used with the same biocatalyst preparation, differences in water solubilities make the enzyme microenvironment quite different. 


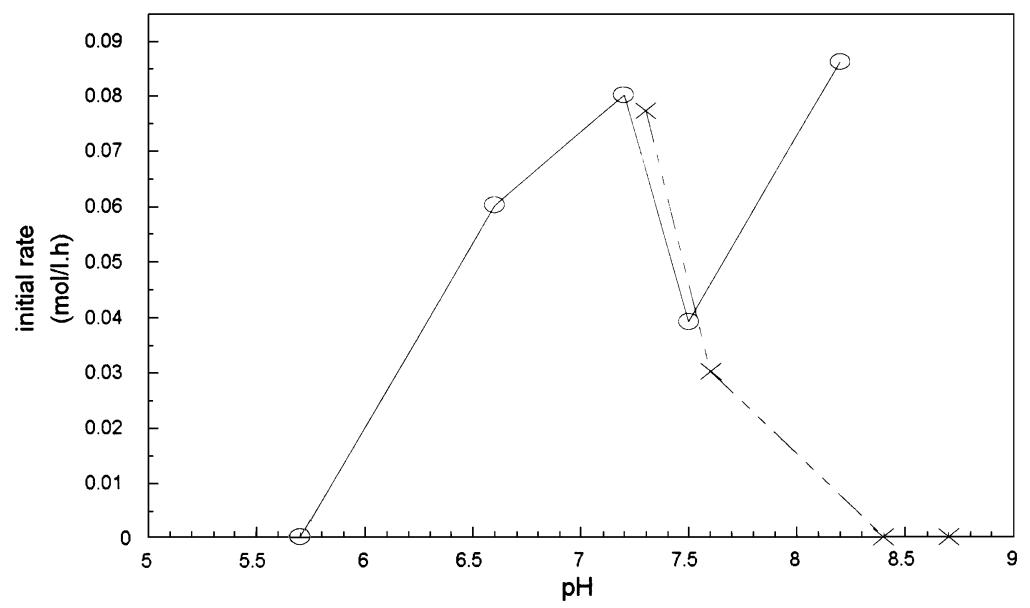

Fig. 3. Influence of $\mathrm{pH}$ of lipase solution, during the immobilisation process, on the esterification activity of the lipolytic preparation: $(-\circ-)$ phosphate buffer; $(-\times-)$ Tris $-\mathrm{HCl}$ buffer.

When a fungal lipase was covalently immobilised onto a graft copolymer and used to catalyse a direct esterification reaction, in the absence of organic solvents, the influence of water activity of both the reaction mixture and the lipolytic preparation on the esterification activity was studied. The immobilised lipase was dehydrated and then equilibrated with an atmosphere of saturated salt solutions to guarantee that the history of hydration was the same. The water mass content of the lipolytic preparation $\left(\mathrm{g} \mathrm{g}^{-1}\right.$ support) was evaluated after the equilibration at each water activity value. Two sets of assays were done under apparently similar conditions and some of the results are shown in Table 1. The profiles of the esterification activity (as well as the ones of the water content of the biocatalyst) were similar until a water activity value of $\approx 0.9$ is reached. After that, when the water activity value was close to one, the initial reaction rate increased with the water content in one set of experiments, but in the other a much larger water content implied a smaller esterification activity. In this last case the water content of the biocatalyst was in excess for the esterification reaction. Comparison with the data from literature did not help very much because we could find esterification activity profiles with both shapes. Each system was a particular case and, sometimes, comparisons should be avoided! In the present case the aggre- gation level of dehydrated particles of biocatalyst before the equilibration with a water saturated atmosphere was not controlled. Fine particles, with average size between 150 and $250 \mu \mathrm{m}$, can be individually dispersed (which is easy to achieve when they are dried or dehydrated) or aggregated (after the last filtration step of the immobilisation process) and, accordingly, have different water contents when equilibrated to the same water activity.

Initially this effect was not considered because an equilibrium is an equilibrium, with all phases reaching the same water activity. However, the aggregation state of powders affects the amount of water retained. When this effect was considered, the dispersed particles retained a larger water mass content and, consequently, a smaller esterification activity. This influence is more remarkable when both the reaction mixture and the lipolytic preparation were equilibrated to a water activity of one. This is an example where the water activity concept alone is not sufficient to describe the microenvironment of the enzyme in terms of available water. Sometimes the real role of water is hidden from a first interpretation.

\subsection{Lipase contact with $n$-hexane}

Organic solvents can induce conformational changes on the lipase molecular structure. One 


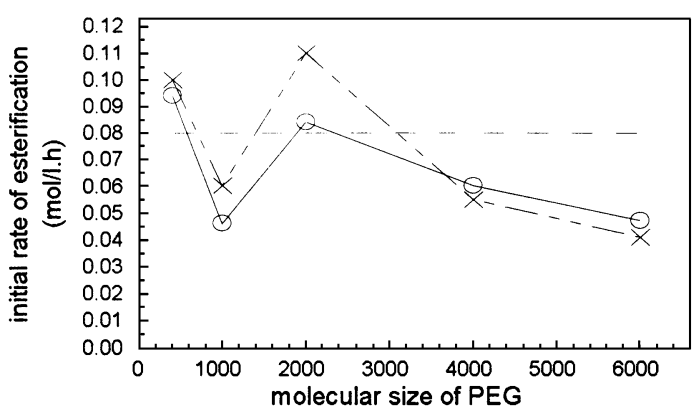

(a)

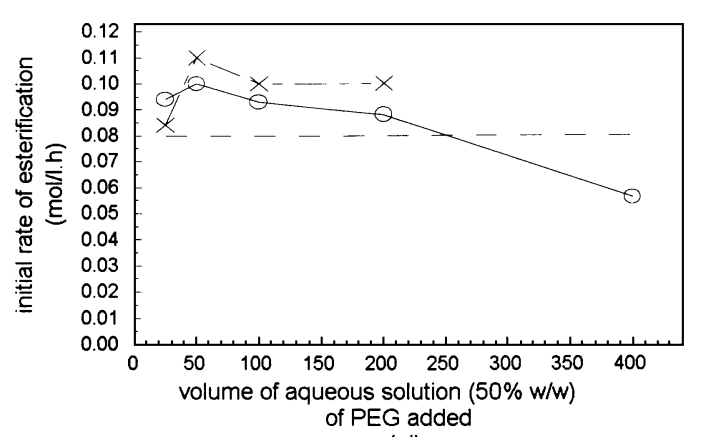

( $\mu$ l)

(b)

Fig. 4. Influence of aqueous PEG $(50 \% \mathrm{w} / \mathrm{w})$ addition to the attachment solution of lipase $(6 \mathrm{ml}$ of phosphate buffer $+200 \mu 1$ of Lipozyme $\left.^{(\mathbb{R}} 10000 \mathrm{~L}\right)$ at the 3rd step of the immobilisation process, on the esterification activity of the lipolytic preparation. The horizontal broken line represents the activity of the immobilised lipase without PEG. (a) Effect of the molecular size of PEG ((- -$)$ $25 \mu \mathrm{l} ;(-\times-) 50 \mu \mathrm{l})$. (b) Effect of the volume of aqueous solution added ((-॰-) PEG 400; $(-\times-)$ PEG 2000).

can take advantage of this, eventually, to induce the surface activation phenomenon, further stabilised by the enzyme immobilisation. Lipase contact with $n$-hexane was established for different time intervals and at different temperatures, both with the free lipase and with the immobilised lipase. In the case of free lipase the contact was made in a water/oil emulsion and the resulting preparation was used in a hydrated state, with $\approx 200 \%(\mathrm{w} / \mathrm{w})$ of water. In the second case the contact was with the dehydrated immobilised lipase, with $\approx 7 \%(\mathrm{w} / \mathrm{w})$ of water; the organic solvent was then removed by filtration and the contacted immobilised lipase was ready for use. The $n$-hexane $(\log P=3.5)$ was selected because it is considered to be a good organic solvent to preserve lipase activity. In Fig. 2a, it can be observed that, after contacting the free lipase in aqueous solution with the $n$-hexane, the activity of the resulting immobilised lipase preparation was decreased. The contribution of water and temperature reinforced the damaging effect of the organic solvent on the free lipase. On the other hand, when the contact of the $n$-hexane was made with the immobilised lipase, no decrease in the activity was observed, as shown in Fig. 2 b.

The stability of the immobilised lipase in the presence of $n$-hexane was greater than in the free state. The stability to organic solvents is characteristic of enzymes that catalyse the transforma- tion of hydrophobic substrates, such as lipases. Immobilisation on solid supports improves the stability to organic solvents and that is another advantage of the immobilisation in biocatalysis in organic medium.

\section{3. $p H$ of lipase solution}

The ionisation state of the active site of lipase molecule must be affected by the type, the concentration and the $\mathrm{pH}$ of buffer salts used in the immobilisation process. Fig. 3 shows that the esterification activity is very sensitive to the $\mathrm{pH}$ of lipase solution during the binding step of the immobilisation process. When lipase was dissolved in water, particles without activity were obtained. The $\mathrm{pH}$ of the lipase solution is a critical factor for the retention of activity of the immobilised preparation, the phosphate buffer $(100 \mathrm{mM})$ at $\mathrm{pH} 7.2$ being a good choice. However, the minimum of the activity profile in the range of $\mathrm{pH}$ between 7.2 and 8.2 could not be explained.

\subsection{Effect of poly(ethylene glycol) as an additive}

In a broad sense, the term 'additives to a reaction mixture' can be used to include the water, the organic solvent and the buffer salts. However, more generally, it is used to indicate the presence 


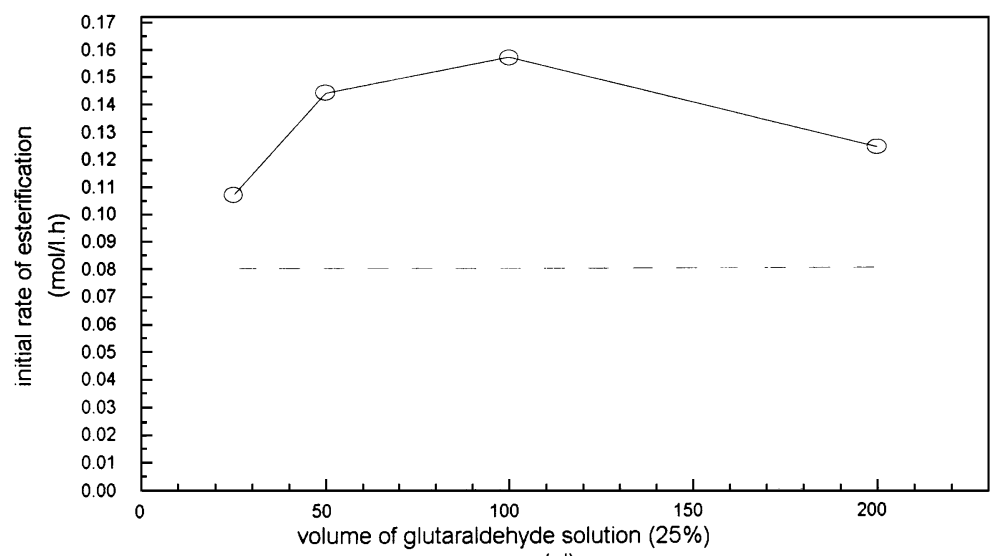

$(\mu \mathrm{l})$

Fig. 5. Effect of the volume of glutaraldehyde solution (25\%) added to lipase solution (6 ml phosphate buffer $+200 \mu 1$ of Lipozyme ${ }^{\mathbb{R}}$ $10000 \mathrm{~L}$ ), when the activation and the immobilisation steps (that is, the 2 nd and 3rd steps of the immobilisation process) were done simultaneously, on the activity of the lipolytic preparation. The broken line represents the activity obtained with the normal three steps immobilisation procedure.

of strange substances in the reaction medium. The effects of these additives on the activity of the lipase preparation are not well understood and, probably, they act by a combination of effects. The kind of additive, its concentration and the contact time are critical parameters that have to be optimised in each case.

The presence of poly(ethylene glycol), (PEG), during the lipase immobilisation process will probably affect the hydration level of the enzyme by modifying the hydrophilicity of the lipase microenvironment. It can be seen from Fig. 4 that the best activation effects were obtained with PEG 400 and PEG 2000 (Fig. 4a), when $50 \mu 1$ of an aqueous solution $(50 \% \mathrm{w} / \mathrm{w})$ (Fig. 4b) were added to the attachment solution of lipase in the 3rd step of the immobilisation process. The decreasing activities obtained with increasing amounts of PEG was interpreted as resulting from increased viscosities.

\subsection{Effect of glutaraldehyde}

The covalent immobilisation was a stepwise process involving the activation of the support and the insertion of a spacer arm in the 1st and 2nd steps and the binding of the lipase. Each step may involve one or more chemical reactions. In these cases the roles that the additives can play are more diverse than in simple physical adsorption immobilisation processes.

The method of immobilisation used in this work was previously described (Rocha et al., 1994) and showed to be very reproducible. When glutaraldehyde, a bifunctional reagent, was added to the lipase solution in the 3rd step of the lipase immobilisation process, the activity of the lipolytic preparation decreased. The fast reaction of its aldehyde groups with the amino groups of the lipase, inhibiting the lipase from binding to the support, could be the explanation for this observation. However, when the method of immobilisation was simplified, by combining the 2 nd and $3 \mathrm{rd}$ steps simultaneously, an increase of $100 \%$ of activity could be observed with much less amount of glutaraldehyde present in the medium, as shown in Fig. 5. In the original procedure the glutaraldehyde was added to activate the support in the $2 \mathrm{nd}$ step and, eventually, some of the glutaraldehyde molecules crosslinked pairs of amino groups of the support, having both aldehyde groups reacted with amino groups of the support; therefore, the activation of the support would be partially inefficient. However, by combining the 2nd and $3 \mathrm{rd}$ steps simultaneously, such a cross-linking would be minimised as some of the glutaraldehyde 
would react preferably and faster with the amino groups of the lipase. Therefore much more aminogroups would be activated in both the support and the lipase and much more lipase would be immobilised with the corresponding increase in the expressed catalytic activity. The quantity of glutaraldehyde added (volume and concentration) to the attachment solution, when both steps were combined, was a critical parameter in enhancing the activity.

\section{Conclusions}

The aggregation level of dehydrated fine particles, before the equilibration with a water saturated atmosphere, affects the water content of the enzyme preparations, and consequently the activity of synthesis.

Lipase immobilisation improves its stability in the presence of organic solvents.

The $\mathrm{pH}$ of lipase solution, during the immobilisation process, is a critical factor.

The addition of small quantities of PEG 400 or PEG 2000 to the attachment solution can enhance the activity of the lipolytic preparation.

The simultaneous activation of lipase and support by glutaraldehyde can also enhance the activity of the lipolytic preparation.

\section{Acknowledgements}

This project was financially supported by the Junta Nacional de Investigação Científica of Lisbon (Project PRAXIS 2/2.1/BIO/34/94), which is greatefully acknowledged.

\section{References}

Adlercreutz, P., 1993. Activation of enzymes in organic media at low water activity by polyols and saccharides. Biochim. Biophys. Acta 1163, 144-148.

Rocha, J.M.S., 1996. PhD thesis, University of Coimbra, Portugal.

Rocha, J.M.S., Gil, M.H., Garcia, F.A.P., 1994. Synthesis of $n$-octyl oleate with lipase from Mucor miehei immobilized onto polyethylene based graft copolymers. Biocatalysis 9, $157-167$.

Triantafyllou, A.O., Wehtje, E., Adlercreutz, P., Mattiasson, B., 1995. Effects of sorbitol addition on the action of free and immobilized hydrolytic enzymes in organic media. Biotechnol. Bioeng. 45, 406-414.

Triantafyllou, A.O., Wehtje, E., Adlercreutz, P., Mattiasson, B., 1997. How do additives affect enzyme activity and stability in nonaqueous media? Biotechnol. Bioeng. 54, 67-76.

Wehtje, E., Adlercreutz, P., Mattiasson, B., 1993. Improved activity retention of enzymes deposited on solid supports. Biotechnol. Bioeng. 41, 171-178.

Yamane, T., Ichiryu, T., Nagata, M., Ueno, A., Shimizu, S., 1990. Intramolecular esterification by lipase powder in microaqueous benzene: factors affecting activity of pure enzyme. Biotechnol. Bioeng. 36, 1063-1069. 